\title{
Saliency detection in complex scenes
}

\author{
Linfeng $\mathrm{Xu}^{*}$, Liaoyuan Zeng, Huiping Duan and Nii Longdon Sowah
}

\begin{abstract}
Detecting multiple salient objects in complex scenes is a challenging task. In this paper, we present a novel method to detect salient objects in images. The proposed method is based on the general 'center-surround' visual attention mechanism and the spatial frequency response of the human visual system (HVS). The saliency computation is performed in a statistical way. This method is modeled following three biologically inspired principles and compute saliency by two 'scatter matrices' which are used to measure the variability within and between two classes, i.e., the center and surrounding regions, respectively. In order to detect multiple salient objects of different sizes in a scene, the saliency of a pixel is estimated via its saliency support region which is defined as the most salient region centered at the pixel. Compliance with human perceptual characteristics enables the proposed method to detect salient objects in complex scenes and predict human fixations. Experimental results on three eye tracking datasets verify the effectiveness of the method and show that the proposed method outperforms the state-of-the-art methods on the visual saliency detection task.
\end{abstract}

Keywords: Visual attention; Saliency model; Complex scene; Human fixation prediction

\section{Introduction}

Visual saliency is a state or quality which makes an item, e.g., an object or a person, prominent from its surroundings. Humans, as well as most primates, have a marvelous ability to interpret complex scenes and pay their attention to the salient objects or regions in the visual environment in real time. Two approaches for the deployment of algorithm based on visual attention have been proposed: the bottom-up and the top-down [1].

For many researches in physiology [2], neuropsychology [3], cognitive science [4], and computer vision [1], it is essential to study the mechanisms of human attention. The understanding of visual attention is helpful for objectof-attention image segmentation [5,6], adaptive coding [7], image registration [8], video analysis [9], and perceptual image/video representation [10]. Most models of attention are bottom-up and biologically inspired. Typically, these models posit that saliency is the impetus for selective vision. Saliency detection can be performed based on center-surround contrast [1], information theory [11,12], graph model [13], common similarity [14-16], or learning methods $[17,18]$.

${ }^{*}$ Correspondence: Ifxu@uestc.edu.cn

School of Electronic Engineering, University of Electronic Science and Technology of China, Xiyuan Avenue 2006, West Hi-Tech Zone, Chengdu, Sichuan 611731, China
The bottom-up saliency detection methods can be broadly classified into local and global schemes. The local contrast-based methods measure the visual rarity with respect to the local neighborhoods using multi-scale image features $[1,19,20]$. The saliency maps generated by such methods usually highlight the object boundary. Furthermore, without knowing the scale of the salient object, the local methods may not detect the salient object accurately. On the contrary, the global contrast-based methods measure the saliency of a pixel by integrating its contrast to all pixels in the image [12,21,22]. Generally, the global methods can generate saliency maps with full resolution and evenly highlighted salient regions. These methods can achieve good accuracy of saliency detection in a scene which consists of a single salient object and a simple background. However, it is hard to detect salient objects from complex scenes due to the global consideration. As shown in Figure 1, the pen container is the most salient according to the fixation density map from the MIT-1003 dataset [23], which shows the region attracting the attention of most subjects. The saliency map in Figure 1c highlights the object boundary, which is obtained from the local method [1]. The global method [22] cannot detect the container because the color of the container is similar to that of the wall and its contrast to all the pixels of the image is low, as shown in Figure 1d.

\section{是 Springer}

(c) 2014 Xu et al.; licensee Springer. This is an Open Access article distributed under the terms of the Creative Commons Attribution License (http://creativecommons.org/licenses/by/2.0), which permits unrestricted use, distribution, and reproduction in any medium, provided the original work is properly cited. 

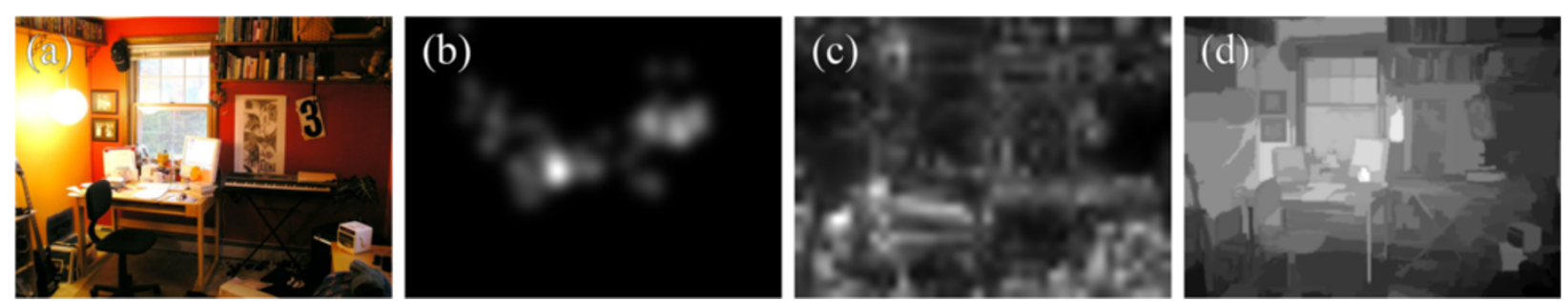

Figure 1 Saliency detection in a complex scene. (a) Original image. (b) Human fixation density map. (c) Saliency map of the local method [1]. (d) Saliency map of the global method [22].

In this paper, we focus on a bottom-up model of saliency detection, which is widely believed to be closely connected to the ubiquity of attention mechanisms in the early stages of biological vision [24]. In neuroscience, numerous studies show that the neuronal response is elevated by the stimulus within the classical receptive field (the center), while stimuli presented in the annular window surrounding the receptive field (the surround) inhibit the response [25]. According to the 'center-surround' mechanisms and the spatial frequency response of the human visual system (HVS) [26], we propose a saliency model based on the following principles of human visual attention:

1. Appearance distinctness between an object and its surroundings. It is generally considered that the response of neurons comes from the contrast of the center region and the surrounding regions [27]. For a pixel $p$ which is inside an object, the pixel is salient if the object is distinct from its surroundings.

2. Unevenness of appearance within an object while appearance similarity within its surrounding region. For a pixel $p$ inside a region (the center), the pixel is salient if the variability of the region is not too low since some intermediate spatial frequency stimuli may evoke a peak response [26]. Meanwhile, the stimuli from the surrounding region should be as weak as possible because the surround is antagonistic to the center and a stronger response will be evoked without the surrounding stimuli [25]. So, the surrounding region should have low variability.

3. Large object size. According to the neuropsychological experiments, attentive response increases when the stimulus size is large and the object is attended [28]. If multiple objects have the same distinctness with respect to their surroundings, humans may pay attention to the larger object first.

These principles reflect the common human characteristics about saliency perception. For example, according to the visual experiments, the spatial frequency response of the HVS, which is similar to the response of a bandpass filter, has a peak response at about 2 to 5 cycles per degree (cpd) and falls off at about $30 \mathrm{cpd}$ [26]. As shown in Figure 2a, a cluttered image is hard to attract people's attention. Principle 2 demands HVS to be sensitive to stimuli from the center and not at the surroundings in order to create stronger center-surround contrast and make the object attentive. In the experiments, we find that each of the principles contributes to the performance of the saliency detector.

Extending our previous work [29], we propose a novel method to measure visual saliency based on biologically plausible saliency mechanisms with a reasonable mathematical formulation. We define the saliency of an image region in a statistical way by means of the scatter matrix. For a pixel in an image, the center region and the surrounding region are defined as the regions centered at the pixel. For a center and a surrounding region, the saliency value is determined by two scatter matrices of the visual features. The first is the 'within-classes scatter matrix' $S_{\mathrm{W}}$ which expresses the similarity between the features of the center region and the surrounding region. The second is the 'between-classes scatter matrix' $S_{\mathrm{B}}$ which describes how the feature statistics in the center region diverge from those in the surrounding region. For a pixel, there exist many concentric regions with different radii, which have different prominence with respect to their surroundings. In order to detect the most salient objects in the scenes, the saliency support region of a pixel is explored, which is the most salient center region among all the concentric center regions. In order to make the large object more salient, the saliency value is weighted using the radius of the saliency support region.

The proposed method has two advantages. First, it is based on the computational architecture of human visual attention. The mechanism of the method is consistent with human perceptual characteristics. So, the method has a good performance for human fixation prediction. Secondly, the proposed method searches the potential saliency support regions to measure the saliency of multiple objects at different scales. This mechanism enables the method to explore various salient objects adaptively. It is effective for saliency detection in complex scenes. 


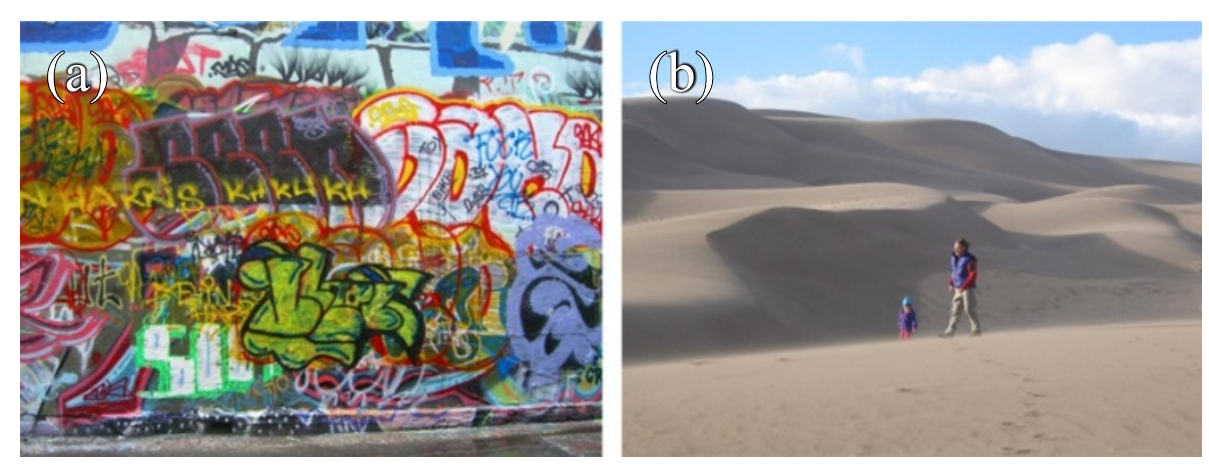

Figure 2 Two types of images. (a) A cluttered image. (b) A concise image. The two people in image (b) attract our attention.

The proposed method is evaluated on three eye tracking datasets which comprise natural images in different scenes and the corresponding human fixation data. Compared to 12 state-of-the-art methods and the human fixation data, the experimental results show that our method outperforms all other methods in terms of receiver operator characteristic (ROC) area metrics for the human fixation prediction task.

This paper is organized as follows: The related work is in Section 2. Section 3 describes the proposed method for saliency detection. The experimental results are provided in Section 4 to verify the effectiveness of the method. Finally, the conclusion is drawn in Section 5.

\section{Related work}

In the past few decades, a lot of bottom-up saliency-driven methods have been proposed in cognitive fields, which can be broadly classified as biologically inspired, purely computational, or an integration of the two [21].

Many attention models are based on the biologically inspired architecture proposed by Koch and Ullman [30], which is motivated from Treisman and Gelade's feature integration theory (FIT) [31]. This structure explains the human visual search strategies, i.e., the visual input is firstly divided into several feature types (e.g., intensity, color, or orientation) which are explored concurrently, and then the conspicuities of the features are combined into a saliency or master map which is a scalar, twodimensional map providing higher intensities for the most prominent areas.

According to this biologically plausible architecture, a popular bottom-up attention model is proposed by Itti et al. [1]. In Itti's model, three multi-resolution extracted local feature contrasts, i.e., luminance, chrominance, and orientation, are mixed to produce a saliency map. Walther and Koch [19] extended Itti's model to infer proto-object regions from individual contrast maps at different spatial scales. These models obtained good results in applications from computer vision to robotics $[19,32]$.
In the last decade, many purely computational methods came up to model saliency with less biological motivation. Ma and Zhang [33] proposed a fuzzy growing method to extract salient objects based on local contrast analysis. Achanta et al. [21] estimated the center-surround contrast by using a frequency-tuned technology. In order to solve the object scale problem, Achanta et al. extended their work by using a symmetric-surround method to vary the bandwidth of the center-surround filtering near image borders [34]. Hu et al. [35] presented a composite saliency indicator and a dynamic weighting strategy to estimate saliency. Hou and Zhang [36] extracted the saliency map from the spectral residual of the log-spectrum of an image. According to the global rarity principle of saliency, Zhai and Shah [37] and Cheng et al. [22] used the histogrambased method to detect the global contrast of a pixel or region. By filtering color values and position values, Perazzi et al. [38] computed the uniqueness and distribution to detect salient regions. Recently, based on a graph-based manifold ranking method, Yang et al. [39] detected saliency of the image elements by ranking the similarity to background and foreground queries. Li et al. [40] performed saliency detection by integrating the dense and sparse reconstruction errors of image regions. These state-of-the-art methods can extract salient regions effectively.

Some of the other methods model saliency based on both of the biological and computational models. Harel et al. [13] used Itti's model to create feature maps, which are integrated into activation maps by using a graphbased approach. Finally, saliency maps are generated by a Markovian algorithm. Bruce and Tsotsos [11] represented the probability distribution of local image patches by using the independent component analysis (ICA). They computed the self-information of image regions to implement a neurally plausible circuit that closely corresponds to visual saliency. Wang et al. [41] use the learned sparse codes to extract some sub-band feature maps which are represented by a random walk-based graph model 
to simulate the information transmission among the neurons.

Besides Bruce's work, some other saliency detectors also established models based on information theory. Itti and Baldi [42] presented a Bayesian definition of surprise to describe saliency. Gao and Vasconcelos [43] proposed a discriminant saliency detection model by maximizing the mutual information of the center and surrounding regions in an image. Klein and Frintrop [44] used the integral histograms to estimate the distributions of the center and surrounding region and expressed the saliency by the Kullback-Leibler divergence (KLD) of these distributions.

Statistical theory has also gotten into the field of saliency detection. Zhang et al. [45] computed saliency based on the self-information of local image features using natural image statistics. Also using natural image statistics, Vigo et al. [46] detected salient edge based on ICA.

We implement the computation of saliency based on the statistics of local image regions. Our work is most closely related to the within-classes scatter matrix and betweenclasses scatter matrix in Fisher's linear discriminant analysis (LDA) which is commonly used for dimensionality reduction before later classification [47]. We use these two scatter matrices to measure the variability within/between the center and surrounding regions which are defined in Section 3.1. Furthermore, we compute the visual saliency based on principles 1 and 2 .

Some methods detect saliency at a single spatial scale $[33,35]$, while others combine feature maps at multiple scales to the final saliency map $[1,19,20]$. Without knowing the scale of the object, these methods may not detect the most salient object accurately. The proposed method finds the saliency support region for a local area and computes the saliency of this region with respect to its surroundings to detect multiple salient objects adaptively.

\section{Proposed method}

In this section, we propose a computational method for saliency detection in images, which is performed in the CIELAB color space. We first define the center region and surrounding region that are used for the centersurround contrast computation. Secondly, a central stimuli sensitivity-based model is proposed to compute the saliency of the center region. Then, the saliency support region of a given pixel is searched to mimic the maximum response of the receptive field in the neurophysiological experiment. Finally, we introduce the visual saliency map generation.

\subsection{Center region and surrounding region}

The saliency computation of the method is based on the selection of two regions, i.e., the center region and surrounding region. According to the center-surround mechanism, the saliency of a pixel is determined by the contrast between the center object (which the pixel belongs to) and the surrounding region. Without a priori information of the center object, we assume that it is approximately within a circular region centered at the pixel, which is referred to as the center region. In this paper, the surrounding region of the center region is defined as the concentric annular region outside the center region, which has the maximal radius toward the nearest image border. For a pixel in the image, there are many center regions. As shown in Figure 3, three center regions for the center pixel of the circles are shown, which are the regions within the blue, purple, or red circles. The corresponding surrounding regions are the annular regions between these circles and the outmost yellow circle.

\subsection{Central stimuli sensitivity-based saliency model}

According to principles 1 and 2, the appearance distinctness between the object and its surrounding and the appearance similarity of each of them are key for visual saliency detection. In order to make an object prominent, the stimuli from the center region should make the HVS sensitive while the stimuli from the surrounding region should not. Following the band-pass characteristic of the spatial frequency response of the HVS [26], we measure the sensitivity in a statistics-theoretic way.

Inspired by the scatter matrices used in Fisher-LDA [47], we use the within-classes scatter matrix to measure the similarity of the center region, $R_{c}$, and the surrounding region, $R_{s}$, which is defined as

$$
S_{\mathrm{W}}=\sum_{n \in\{1,2\}} \sum_{p \in R^{n}}\left(x_{p}-\mu_{n}\right)\left(x_{p}-\mu_{n}\right)^{T}
$$

where $R^{1}$ denotes the region $R_{c}, R^{2}$ denotes the region $R_{s}$, $x_{p}$ is the feature vector of pixel $p$ (the vector contains the intensity and color features in the experiments), and $\mu_{n}$ is the mean feature vector of the region $R^{n}$. The matrix of each region is normalized by the number of the pixels in the region. The eigenvalues of the scatter matrix are related to the spatial frequency of the region. If a region is flat, the pixels in the region concentrate on their mean. In other words, the pixels in the region are not scattered. For two flat regions, the eigenvalues of $S_{\mathrm{W}}$ are small. According to principle 2, we define the sum of the eigenvalues of $S_{\mathrm{W}}$ to be inversely proportional to the saliency value in the method. However, a flat center region with low frequency may get a large saliency value, which violates principle 2 . In order to measure the sensitivity to the center stimuli, which has a peak response at an intermediate frequency [26], we modify (1) by weighting the matrix of $R_{c}$, which can be represented as

$$
S_{\mathrm{W}}=\omega_{c} \sum_{p \in R^{1}}\left(x_{p}-\mu_{1}\right)\left(x_{p}-\mu_{1}\right)^{T}+\sum_{p \in R^{2}}\left(x_{p}-\mu_{2}\right)\left(x_{p}-\mu_{2}\right)^{T}
$$



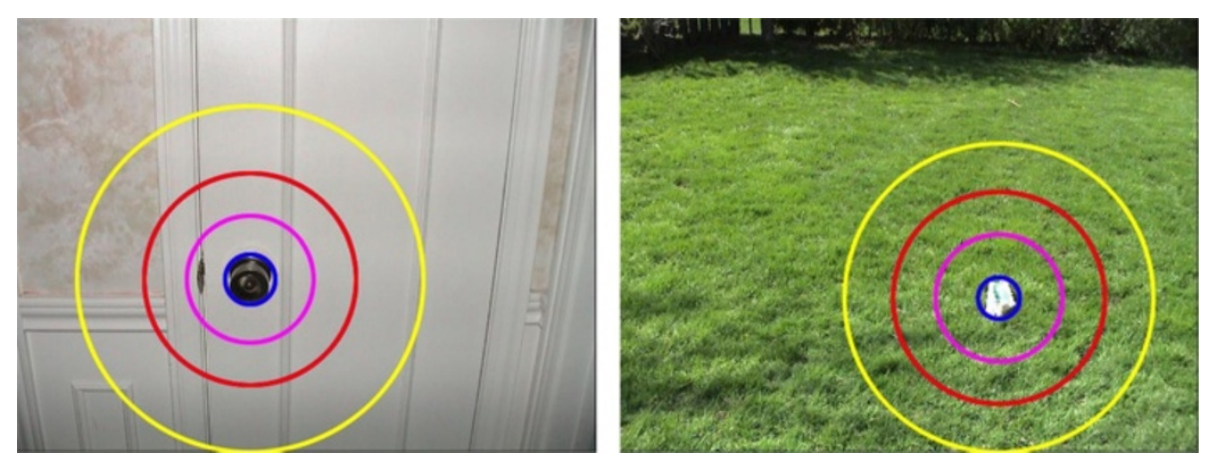

Figure 3 Examples of center regions and surrounding region. Three center regions for the pixel in the circle center are shown, which are the regions within the blue, purple, or red circles. The corresponding surrounding regions are the annular regions between these circles and the outmost yellow circle.

where $\omega_{c}$ is a empirically set parameter to control the contribution of the similarity of $R_{c}$ to the computed saliency value. By setting $\omega_{c}$ to 1 , a flat center region may produce a large saliency value. If $\omega_{c}$ is decreased, an uneven center region with a higher spatial frequency may generate a large saliency value. We will demonstrate in Section 4.5 that the weight $\omega_{c}$ plays a significant role in the saliency computing.

To measure the difference of $R_{c}$ and $R_{s}$, the betweenclasses scatter matrix is used, which is defined as

$$
S_{\mathrm{B}}=\sum_{n \in\{1,2\}}\left(\mu_{n}-\mu\right)\left(\mu_{n}-\mu\right)^{T}
$$

where $\mu$ is the overall mean feature vector of the pixels in $R_{c} \bigcup R_{s}$. For two regions which are distinct from each other, the eigenvalues of $S_{\mathrm{B}}$ are large.

The saliency of a particular center region depends on the traces of $S_{\mathrm{W}}$ and $S_{\mathrm{B}}$, i.e., the sums of eigenvalues of the two scatter matrices, which is computed by

$$
\operatorname{Sal}\left(R_{c}\right)=\frac{\operatorname{trace}\left(S_{\mathrm{B}}\right)}{\operatorname{trace}\left(S_{\mathrm{W}}\right)} .
$$

A center region which is distinct from its flat surrounding region has a high saliency value.

\subsection{Saliency support region}

As shown in Figure 3, many center regions exist for a pixel. Some of them are salient, such as the region within the blue circle, while some others are not, such as the regions within the red and purple circles. As mentioned in Section 2, some of the previous work preset multiple spatial scales or use a single scale to detect saliency, which may fail to find the salient object.

According to the spatial summation curves in the neurophysiological experiments, when the visual stimuli cover the area of receptive field center, the neural responses reach the peak [48]. We believe that for a salient object, there exists a support to form the saliency quality, which generates a peak response in the neuron. We attempt to find the support region which generates the most intensive saliency with respect to its surrounding region, referred to as the saliency support region.

We define the saliency support region of a pixel as the center region which has the largest saliency value using (4). The saliency support region, SSR, can be formulated as

$$
\mathrm{SSR}=\arg \max _{R_{c} \in \mathbb{A}} \operatorname{Sal}\left(R_{c}\right)
$$

where $\mathbb{A}$ is the set of all the possible center regions of a pixel. As shown in Figure 4, the saliency support region of the pixel in the middle of the flower is the region within the red circle, which consists of the stamens and has the largest saliency value with respect to its surrounding region of flower petals and green leaves. Other center regions are less salient than the saliency support region. We use the saliency value of the saliency support region to represent the saliency of the center pixel of the SSR. The exploration of the saliency support region intends to measure the maximal saliency of pixels and find salient objects with different sizes. For a pixel, there are many possible center regions that need to be compared. In order to reduce computational expense, we reduce the candidate center regions by sampling their radii at a fixed interval in the implementation, i.e., only the regions with the sampled radii are compared.

\subsection{Visual saliency map}

In the saliency map of the proposed method, the value of a pixel is determined by the saliency value of its saliency support region. According to the principle 3, large objects may attract more human attention than small details. For example, the large desk lamp in Figure 5 is more attentive than the small lights. In terms of saliency support region, if the most salient region is large, it may attract more attention. In the method, the saliency value is weighted 


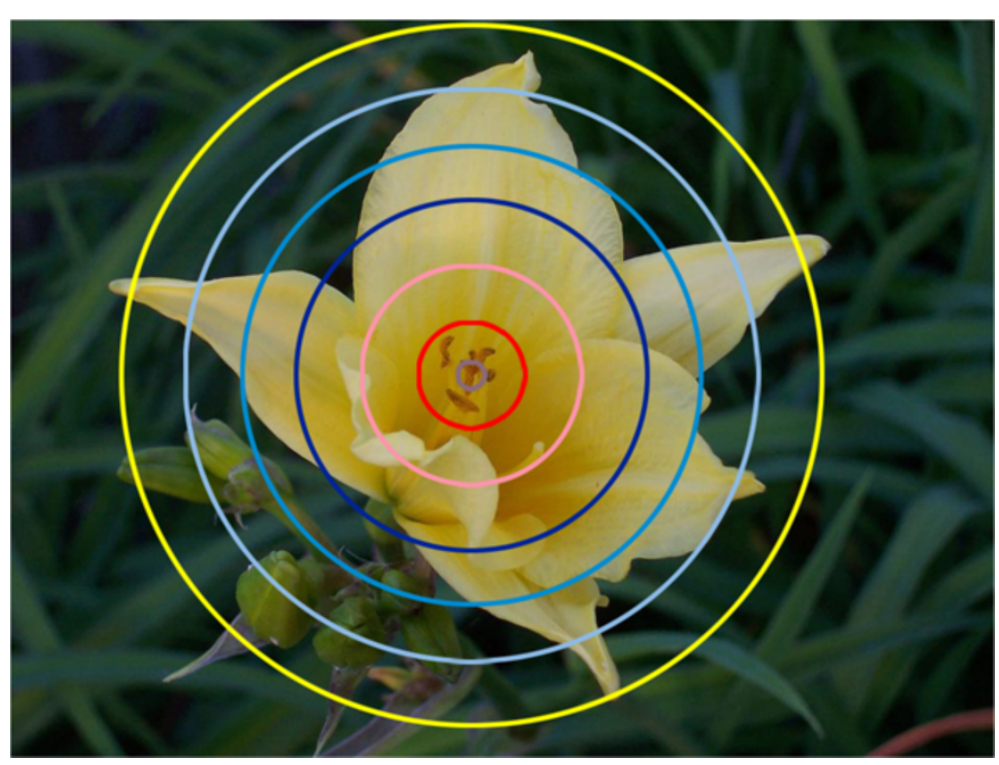

Figure 4 An example of the saliency support region. The region within the red circle is the saliency support region of the pixel in the middle of flower stamen, which has the largest saliency value with respect to its surrounding region of flower petals and green leaves.

by the radius of the saliency support region, which can be represented as

$$
\operatorname{Sal}(p)=\operatorname{Sal}(\mathrm{SSR}) \cdot r(\mathrm{SSR})
$$

where $p$ is the center pixel of the saliency support region (SSR), and $r$ (SSR) denotes the radius of SSR.

Instead of measuring the saliency values of all the pixels, we sample the pixels at an interval (e.g., 10 pixels) for computation reduction. The lattice of the sampled pixels is interpolated bilinearly and Gaussian filtered with $\sigma=25$ to generate the final saliency map of the image, as shown in Figure 6.

\section{Experiments}

In this section, we apply the proposed method on three public eye tracking datasets (two color image datasets and one gray image dataset) to evaluate the performance of human fixation prediction. These datasets comprise natural images, containing different objects and scenes, and the corresponding human fixations. The proposed

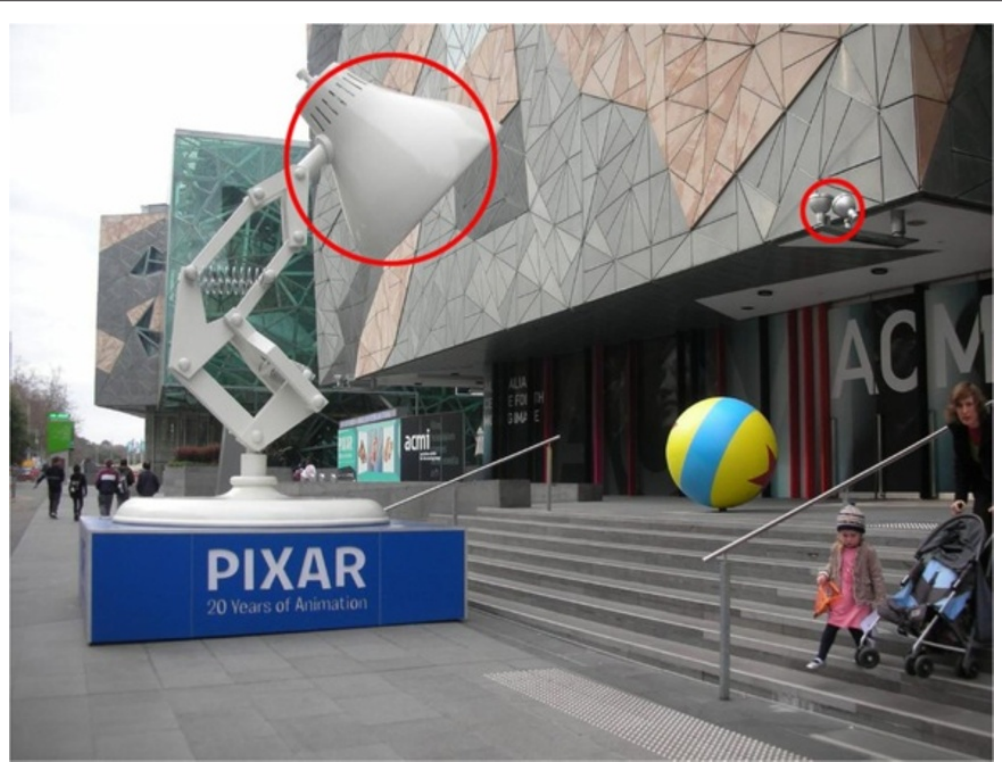

Figure 5 An example of large object size for saliency detection. The large desk lamp may attract more attention than the small lamps. 

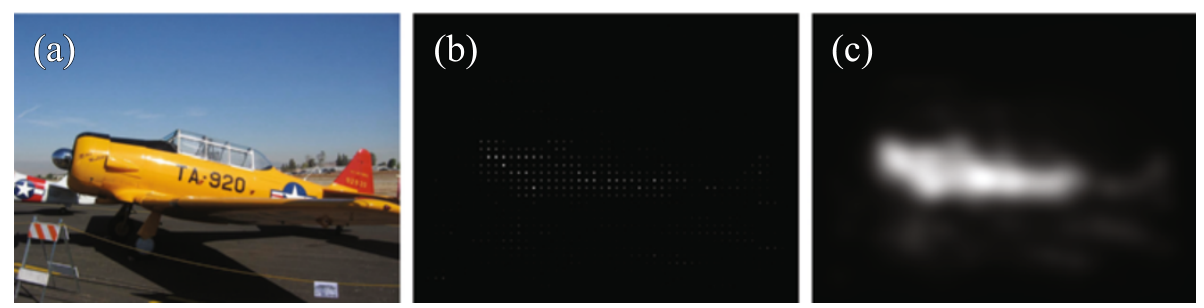

Figure 6 An example of the saliency map. (a) Original image. (b) Lattice of the sampled pixels. (c) Saliency map generated by interpolation and filtering.

method is compared with the state-of-the-art bottomup methods based on a well-known validation approach. The qualitative and quantitative assessments of detection results are reported.

\subsection{Parameter setting}

There is a parameter in the proposed method: the weight $\omega_{c}$ of the within-classes scatter matrix of the center region in (2). We set the weight $\omega_{c}=0.1$ because it can obtain large areas under the ROC curves in the experiments on the three datasets. The relationship between the parameter and the performance of the method is discussed in Section 4.5.

\subsection{Experiments on BRUCE color image dataset}

In the first experiment, we perform saliency computations on the popular color image dataset introduced by Bruce and Tsotsos [11], which consists of 120 images in indoor and outdoor scenes, such as human objects, furniture, phones, fruits, cars, buildings, streets, etc. All the image sizes are $681 \times 511$ pixels. In the dataset, 20 subjects' fixations are recorded for each image. To compare the saliency maps with the human fixations objectively, we use the popular validation approach as in [11]. The area under the ROC curve is used to quantitatively evaluate the performance of visual saliency detection.

We compare the proposed method with 12 state-ofthe-art bottom-up saliency detection methods, i.e., Itti's model (IT) [1], attention information maximization (AIM) [11], spectral residual (SR) [36], graph-based visual saliency (GB) [13], site entropy rate (SER) [41], context aware (CA) [49], salient region detection (AC) [20], maximum symmetric surround (MSS) [34], region-based contrast (RC) [22], saliency filters (SF) [38], graph-based manifold ranking (MR) [39], and dense and sparse reconstruction (DSR) [40] which are listed in Table 1. These methods involve a variety of saliency models, such as biologically motivated (e.g., IT), computational (e.g., AC and MSS), frequency-based (e.g., SR), mixed (e.g., AIM and $\mathrm{GB}$ ), local contrast (e.g., IT and AC), global contrast (e.g., RC), and state-of-the-art (e.g., SF, MR, and DSR) models. Some of the methods are used to predict human fixations, such as AIM, GB, and SER, and some others show excellent performance in the salient region/object extraction task, such as RC, SF, MR, and DSR. For all these methods, we use the source codes or executable codes by the authors. The proposed method is implemented in Matlab.

Figure 7 qualitatively shows the comparison results of saliency maps for some test images of the BRUCE dataset. The original images are shown in Figure $7 \mathrm{a}$, while the maps obtained from the state-of-the-art methods are given in Figure $7 b, c, d, e, f, g, h, i, j, k, l, m$, respectively. The results of the proposed method are shown in Figure $7 \mathrm{n}$. The fixation density maps are shown in the final column, which are generated from the sum of 2D Gaussians corresponding to each fixation point of all the subjects [11]. It can be seen that most of the methods can detect the single salient object in a simple scene, such as the second row. However, it is challenging for the images which

Table 1 The state-of-the-art methods

\begin{tabular}{lll}
\hline Algorithm name & Reference & Implementation code \\
\hline Itti's model (IT) & Itti [1] & Matlab code by Harel [13] \\
$\begin{array}{l}\text { Attention information } \\
\text { max. (AIM) }\end{array}$ & Bruce [11] & Matlab code by author \\
$\begin{array}{l}\text { Spectral residual (SR) } \\
\text { Graph-based visual } \\
\text { saliency (GB) }\end{array}$ & Hou [36] & Matlab code by author \\
$\begin{array}{l}\text { Site entropy rate (SER) } \\
\text { Context aware (CA) }\end{array}$ & Wang [41] & Matlab code by author \\
$\begin{array}{l}\text { Salient region } \\
\text { detection (AC) }\end{array}$ & Achanta [20] & Executable code by author \\
$\begin{array}{l}\text { Maximum symmetric } \\
\text { surround (MSS) }\end{array}$ & Achanta [34] & Executable code by author \\
$\begin{array}{l}\text { Region-based } \\
\text { contrast (RC) }\end{array}$ & Cheng [22] & Executable code by author \\
Saliency filters (SF) & Perazzi [38] & C code by author \\
$\begin{array}{l}\text { Graph-based } \\
\text { manifold ranking (MR) }\end{array}$ & Yang [39] & Matlab code by author \\
$\begin{array}{l}\text { Dense and sparse } \\
\text { reconstruction (DSR) }\end{array}$ & Li [40] & Matlab code by author \\
\hline
\end{tabular}




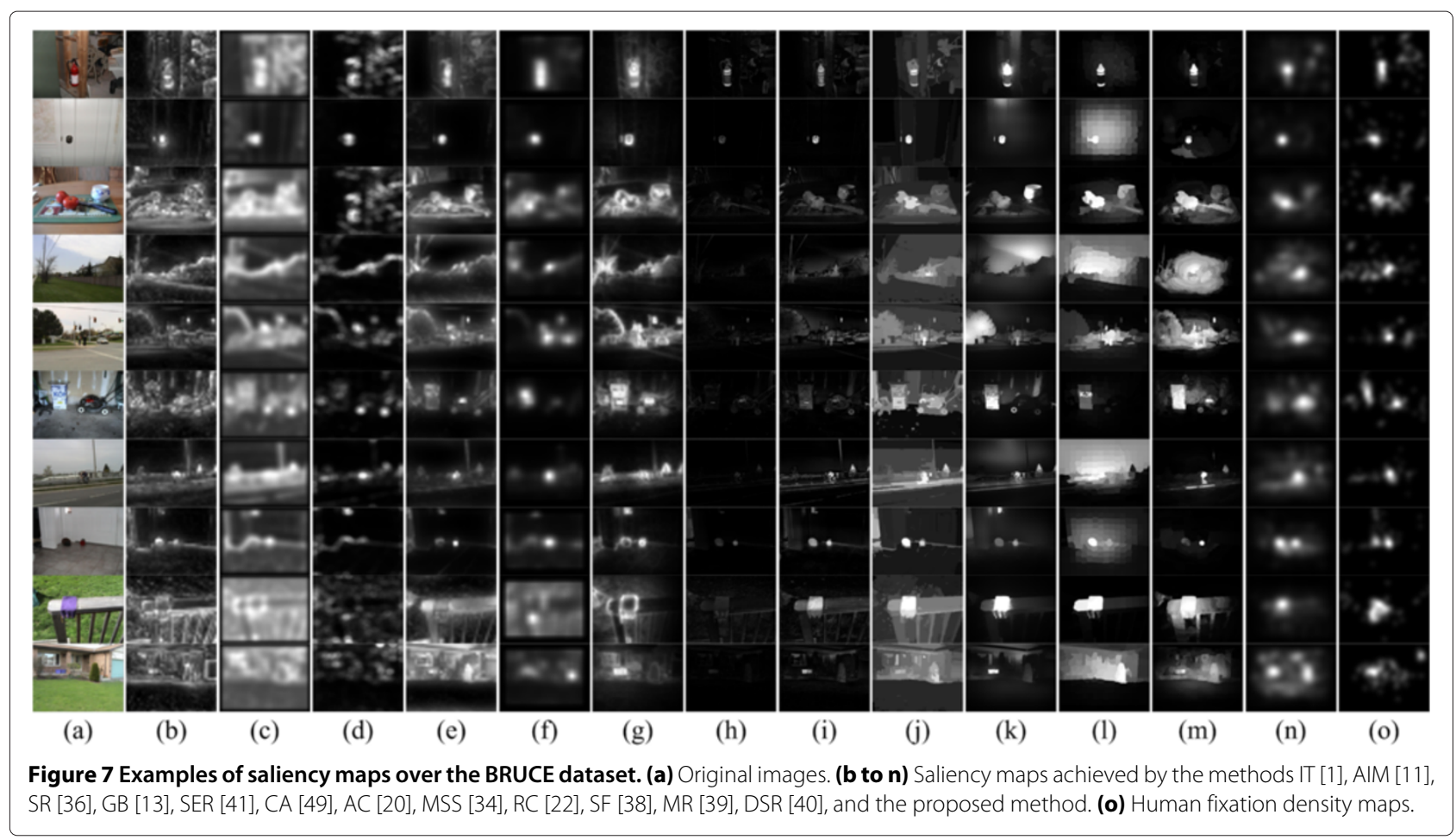

consist of multiple objects or complex scenes. The comparison results show that our maps which are based on the biological perception mechanisms are more consistent with the human fixation density maps. For example, in the third image, the scene is a bit complex. There are some objects, e.g., the tomatoes, cup, and knife, on a motley table mat. The two tomatoes have the stronger local contrast than the other objects. As a result, it is shown from the fixation density map that the tomatoes attract the attention of most of the subjects. However, most of the methods fail to do well. In the methods IT, AIM, SR, GB, and $C A$, saliency detection is performed by searching the high-frequency regions. So, the boundaries of the objects are detected as the most salient. In some methods, such as SER, MSS, and SF, the pixels in the cup, knife, or table mat are very salient due to the global consideration. The proposed method searches the saliency support region to explore the most salient region. So, the tomatoes are assigned the highest saliency value and recognized as the most salient region. Meanwhile, if multiple salient objects have the comparable local contrast, our method also can detect these objects. For example, for the eighth image, the two salient objects are detected by the proposed method.

In order to evaluate the quality of the proposed method, we perform a quantitative comparison by computing the salient degree between the extracted saliency map and the human fixations. The popular validation approach, the ROC area [11], is used to evaluate the performance of visual saliency detection. The results of ROC areas of the compared methods on this dataset are shown in the second column of Table 2. Among the existing methods, the very recent method MR has the best fixation prediction performance on this dataset, whose ROC area is 0.7378 . It shows that MR does well not only in the salient region detection task but also in this fixation prediction evaluation. However, it can be seen that the ROC

Table 2 The ROC areas on three eye tracking datasets

\begin{tabular}{lccc}
\hline Method & $\begin{array}{c}\text { BRUCE } \\
\text { dataset }\end{array}$ & $\begin{array}{c}\text { MIT-1003 } \\
\text { dataset }\end{array}$ & $\begin{array}{c}\text { DOVES } \\
\text { dataset }\end{array}$ \\
\hline IT [1] & 0.5709 & 0.6835 & 0.5548 \\
AIM [11] & 0.6275 & 0.7662 & 0.6201 \\
SR [36] & 0.5315 & 0.6977 & 0.5429 \\
GB [13] & 0.5237 & 0.6857 & 0.5061 \\
SER [41] & 0.6632 & 0.7835 & 0.6716 \\
CA [49] & 0.6307 & 0.7585 & 0.6271 \\
AC [20] & 0.5520 & 0.6251 & 0.5312 \\
MSS [34] & 0.6107 & 0.6774 & 0.5530 \\
RC [22] & 0.6461 & 0.7568 & 0.6176 \\
SF [38] & 0.6601 & 0.7019 & 0.6492 \\
MR [39] & 0.7378 & 0.7766 & 0.7375 \\
DSR [40] & 0.7144 & 0.7908 & 0.7021 \\
PM ${ }^{a}$ & 0.7626 & 0.8027 & 0.7503 \\
\hline
\end{tabular}

a The proposed method. The numbers in italics show the best method which achieves the maximal ROC area on each dataset. 
area of the proposed method is about 0.025 (3.4\%) higher than MR. It demonstrates that the proposed method outperforms the 12 state-of-the-art methods on predicting human fixations on this eye tracking dataset.

The results listed in Table 2 are different from some of the reported results $[11,13,41]$. In the existing comparison methods, the fixation mask is obtained by setting a quantization threshold, i.e., the threshold classifies the locations in a fixation density map into fixations and nonfixations. So, different quantization thresholds lead to different results. To perform a fair comparison, we use the fixation points provided by the dataset as the ground truth for all the compared methods, i.e., only the points are fixations and the rest are non-fixations. The ROC areas of the compared methods are generated using the Matlab code provided by Harel et al. [13].

\subsection{Experiments on MIT-1003 color image dataset}

We perform saliency computations on another color image dataset introduced by Judd et al. [23]. The MIT1003 dataset contains 1,003 natural images of varying dimensions (the maximal dimension of the width and height is 1,024 pixels), along with human fixation data from 15 subjects. The images in this dataset contain different scenes and objects, as well as many semantic objects, such as faces, people, body parts, and text, which are not modeled by bottom-up saliency [23].

We compare the proposed method with the same 12 methods listed in Table 1 on this dataset. Comparison results of saliency maps for some of the test images are shown in Figure 8. The original images are shown in Figure 8a, while the results from the state-of-the-art methods are given in Figure 8b,c,d,e,f,g,h,i,j,k,l,m, respectively. The results of the proposed method are shown in Figure $8 \mathrm{n}$, and the fixation density maps provided in the dataset are given in Figure 8o. For the fifth and seventh images that contain complex background, the global contrast-based methods, e.g., RC and SF, are apt to highlight the noise regions, e.g., shadows, and overlook the key regions. Some local contrast-based methods, such as IT, AIM, SR, GB, and CA, detect the boundary of objects as the most salient like their performance on the BRUCE dataset. Although some methods preset multiple scales, such as IT and AC, they cannot effectively detect salient objects in the complex scenes. Method SER performs better than other existing methods. However, for the images with multiple objects, such as the sixth and seventh images, SER only detects one object. By following the biological perception mechanisms and exploring the saliency support region, the proposed method can achieve good performance to predict most of the fixations in the images that contain complex scenes and semantic objects. For example, in the fifth image, the proposed method detects the face of the girl as the most salient, which is consistent with most of the subjects. However, most of the other methods fail to find the face of the girl and detect the boundaries of different objects. Another example is the sixth and seventh images, in which the proposed method detects two salient people due to the saliency support region exploration.

The results of ROC areas of the compared methods on this dataset are shown in the third column of Table 2. Among the existing methods, the very recent method DSR shows the best performance on this dataset. The proposed method achieves a slightly higher ROC area than DSR and also outperforms the state-of-the-art methods on this human fixation dataset. We notice that the improvement of our method on this dataset is not as overt as on the BRUCE dataset. The main reason is that the MIT-1003 dataset contains many semantic objects which put forward challenges to the bottom-up models. The detected results by our method are based on the bottomup contrast, which may diverge from the fixations of the subjects. Using some high-level features may improve the results.

\subsection{Experiments on DOVES gray image dataset}

In the third experiment, we test the proposed method on a gray image dataset, DOVES, which is introduced by van der Linde et al. [50]. The DOVES dataset contains 101 natural images and the eye tracking data from 29 subjects. All the image sizes are $1,024 \times 768$ pixels. Because the first fixations of each eye movement trace of the subjects are forced at the center of the image [50], these fixations are removed in the experiments.

We also compare the proposed method with the 12 methods listed in Table 1 on the DOVES dataset. However, some of the methods are not compatible with gray images. For the methods AIM, CA, AC, and MSS, we use the RGB images whose three components are generated by duplicating the intensity of the gray images. Comparison results of saliency maps for some test images from this gray image dataset are shown in Figure 9. The original images are shown in Figure 9a, while the maps from the 12 previous methods are shown in Figure $9 \mathrm{~b}, \mathrm{c}, \mathrm{d}, \mathrm{e}, \mathrm{f}, \mathrm{g}, \mathrm{h}, \mathrm{i}, \mathrm{j}, \mathrm{k}, \mathrm{l}, \mathrm{m}$, respectively. The results of the proposed method are given in Figure $9 n$ and the human fixation density maps are shown in the final column. It can be seen that the cue of colors is key for most of the existing methods. For these gray images, most of the methods detect the bright sky or dark shadows as the salient regions, and method MR generally detects the image center as the salient region. However, the proposed method is less sensitive to the lack of colors, which mainly depends on the local statistical property of the image. The saliency maps generated by the proposed method are consistent with 


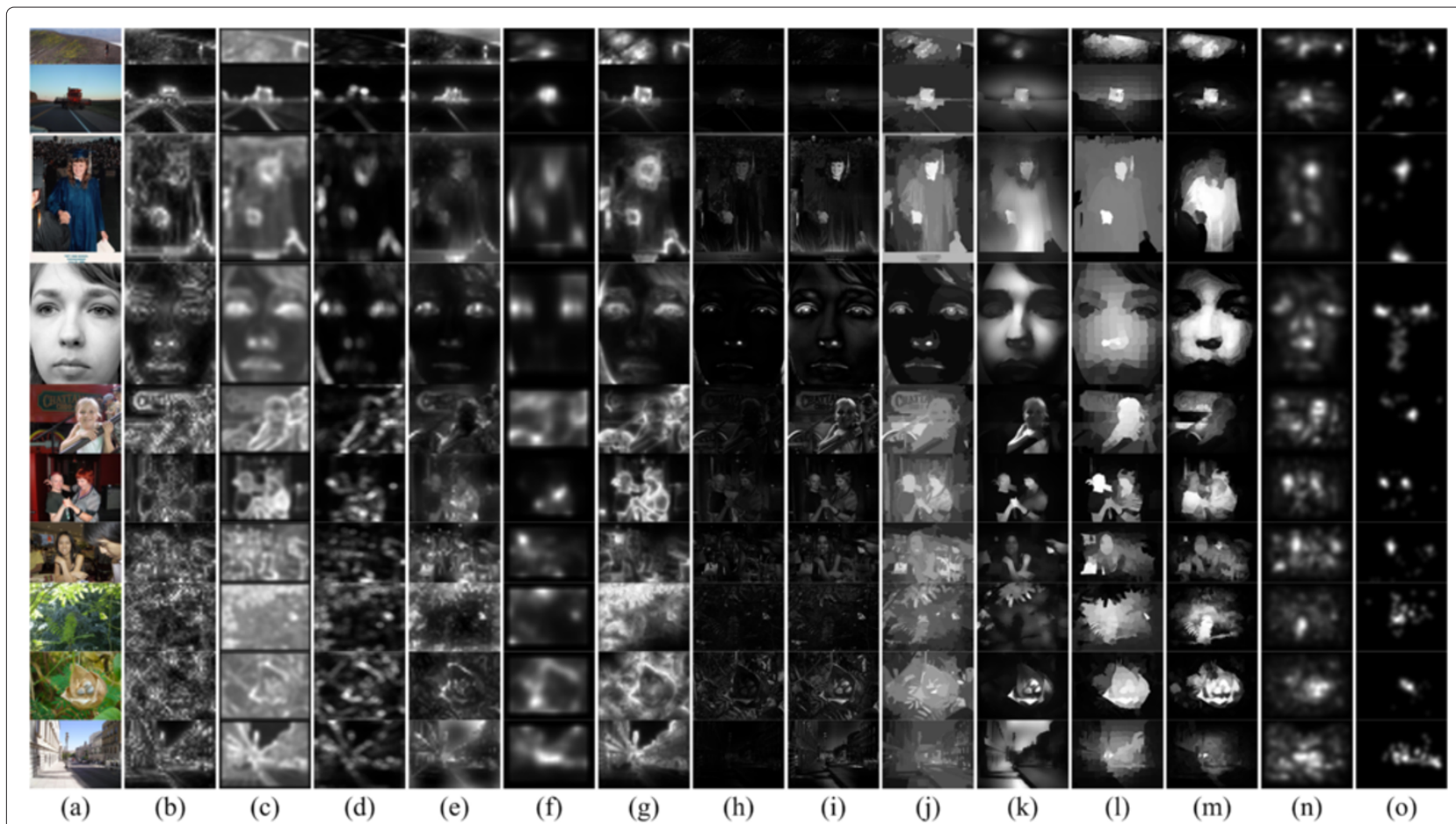

Figure 8 Examples of saliency maps over the MIT-1003 dataset. (a) Original images. (b to $\mathbf{n}$ ) Saliency maps achieved by the methods IT [1], AIM [1 1], SR [36], GB [13], SER [41], CA [49], AC [20], MSS [34], RC [22], SF [38], MR [39], DSR [40], and the proposed method. (o) Human fixation density maps.

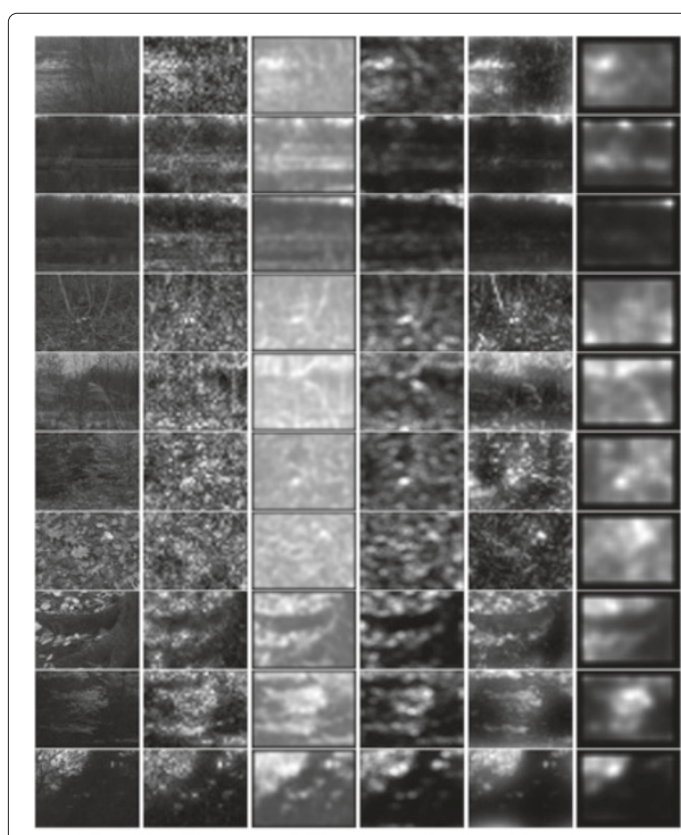

(a)

(b)

(c)

(d)

(e)

(f)

(g)

(h)

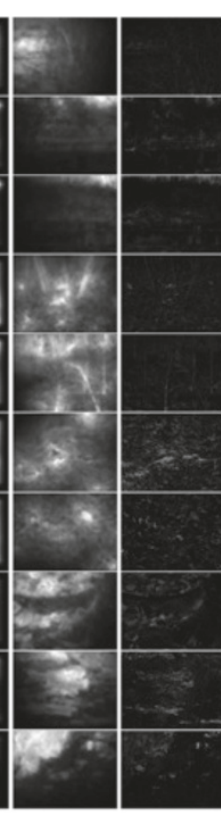

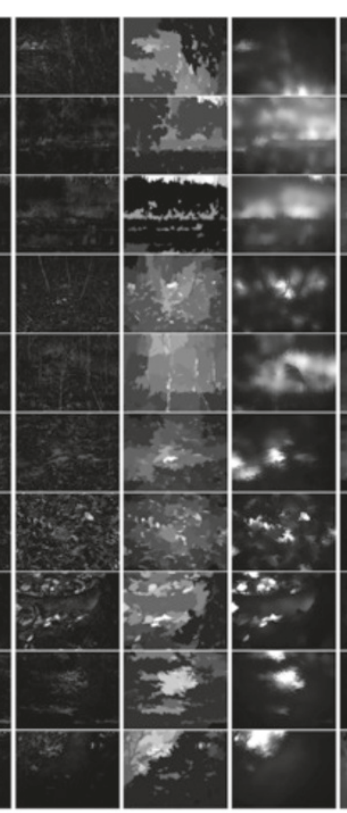

(i)

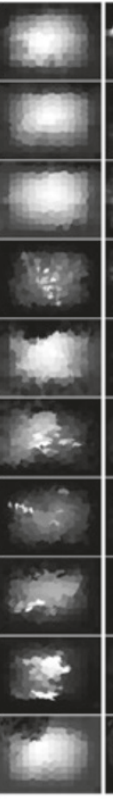

(1)

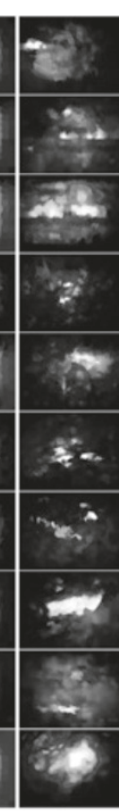

(m)

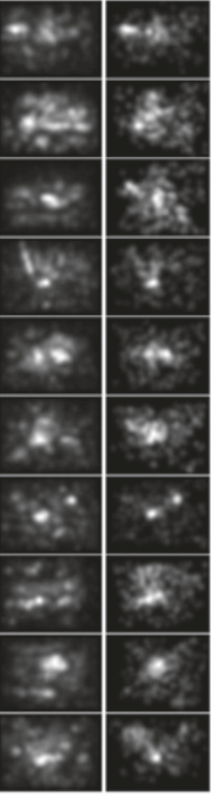

(n)

(o)

Figure 9 Examples of saliency maps over the DOVES dataset. (a) Original images. (b to $\mathbf{n}$ ) Saliency maps achieved by the methods IT [1], AIM [11], SR [36], GB [13], SER [41], CA [49], AC [20], MSS [34], RC [22], SF [38], MR [39], DSR [40], and the proposed method. (o) Human fixation density maps. 


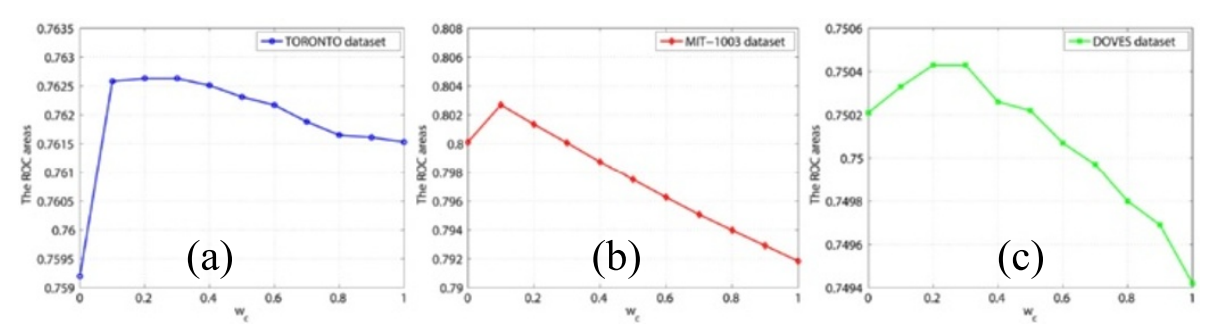

Figure 10 The relationship between the ROC area and the weight $\omega_{\boldsymbol{c}}$. Results on (a) the BRUCE dataset, (b) the MIT-1003 dataset, and (c) the DOVES dataset.

human fixations well. It shows that the proposed method has a good ability to predict fixations even for gray images.

The results of ROC area of all the compared methods on the DOVES dataset are presented in the fourth column of Table 2. Method MR shows the highest ROC area (0.7375) on this dataset compared to the previous methods. The main reason is that most of the subjects tend to focus their fixations on the image center if there are no very prominent regions. Compared with MR, the proposed method achieves about $2 \%$ improvement of ROC area. It shows that the proposed method outperforms the state-of-the-art methods on fixation prediction for gray images.

\subsection{Discussion}

In our model, the parameter $\omega_{c}$ is designed to determine the region of which frequency will be assigned a high saliency value. If $\omega_{c}$ is set to 1 and 0 , the regions with very low and high frequency will be assigned high saliency values, respectively. According to the HVS principles, the very low and high frequency regions may weaken the response of the HVS. So, an inappropriate value of $\omega_{c}$ will lead to the wrong detection, i.e., the ROC area may be small.

Figure 10 shows the relationship between the ROC area and the weight $\omega_{c}$ in (2) on the three datasets. Generally, when the weight $\omega_{c}$ is bigger than 0.3 , the ROC area decreases as the weight increases. That is to say, the salient objects are not necessarily flat according to the evaluation based on the human fixation data. On the contrary, if no constraints are imposed on the similarity of the center region, i.e., $\omega_{c}=0$, the ROC area drops, especially on the BRUCE dataset, the ROC area in Figure 10a is the lowest when $\omega_{c}=0$. That is to say, if the center region is rather cluttered, it may not attract human attention. This result is consistent with the spatial frequency response of the HVS [26]. The curves in Figure 10 are similar to the mirror of the spatial frequency response curve, which show that the response reaches a maximum when the spatial frequency gets an intermediate frequency, $\omega_{c}$ is between 0.1 and 0.3 , while it falls off rapidly at higher frequency, namely $\omega_{c}=0$. The response decreases slowly as the frequency decreases to $\mathrm{DC}$, i.e., $\omega_{c}=1$, from the intermediate frequency. It is worth noting that our model is biologically plausible. It is difficult to denote the spatial frequency by specific values of $\omega_{c}$.

The performance improvement of the proposed method in the fixation prediction experiments verifies the effectiveness of the scatter matrix-based saliency computation and the saliency support region exploration. However, since we use the pixel-wise processing manner and the SSR is searched for every processed pixel, the method is computationally expensive. We therefore adopt the subsampling method to reduce the cost. The average running time on the BRUCE dataset to generate the saliency map is $60.63 \mathrm{~s}$ when measured on an Intel $3.20-\mathrm{GHz}$ CPU with 3-GB RAM in Matlab implementation. In the future, we will study the superpixel-based processing to make the algorithm more efficient.

\section{Conclusions}

In this paper, we propose a novel method to compute visual saliency in a statistical way. According to three principles of human visual attention, we use the withinclasses scatter matrix and the between-classes scatter matrix to measure the similarity and distinctness within and between the center region and the surrounding region, respectively. Furthermore, the saliency of the center region is computed by the two scatter matrices. In order to detect the salient objects with different sizes, the saliency support region is explored and the saliency value of the center pixel of the region is obtained. To make the large object more salient, the saliency value is weighted by the radius of the saliency support region. Experimental results are obtained by applying the proposed method to three eye tracking datasets. The results show that the proposed method outperforms the state-of-the-art methods on saliency detection in complex scenes and human fixation prediction. 


\section{Competing interests}

The authors declare that they have no competing interests.

\section{Acknowledgements}

This work was partially supported by NSFC (No. 61201274), National High Technology Research and Development Program of China (863 Program, No. 2012AA011503), and Fundamental Research Funds for the Central Universities (No. ZYGX2012J025).

Received: 27 July 2013 Accepted: 17 June 2014

Published: 24 June 2014

\section{References}

1. L Itti, C Koch, E Niebur, A model of saliency-based visual attention for rapid scene analysis. IEEE Trans. Pattern Anal. Mach. Intell. 20(11), 1254-1259 (1998)

2. S Kastner, LG Ungerleider, Mechanisms of visual attention in the human cortex. Ann. Rev. Neurosci. 23, 315-341 (2000)

3. HE Egeth, $\mathrm{S}$ Yantis, Visual attention: control, representation, and time course. Ann. Rev. Psychol. 48, 269-297 (1997)

4. R Desimone, J Duncan, Neural mechanisms of selective visual attention. Ann. Rev. Neurosci. 18, 193-222 (1995)

5. H Li, KN Ngan, Saliency model based face segmentation in head-and-shoulder video sequences. J. Vis. Commun. Image Represen. 19(5), 320-333 (2008)

6. H Li, KN Ngan, Learning to extract focused objects from low DOF images IEEE Trans. Circuits Syst. Video Technol. 21(11), 1571-1580 (2011)

7. KC Liu, Prediction error preprocessing for perceptual color image compression. EURASIP J. Image Video Process. 2012, 3 (2012)

8. D Mahapatra, Y Sun, Rigid registration of renal perfusion images using a neurobiology-based visual saliency model. EURASIP J. Image Video Process. 2010, 195640 (2010)

9. J You, G Liu, A novel attention model and its application in video analysis. Appl. Math. Comput. 185(2), 963-975 (2007)

10. M Mancas, B Gosselin, B Macq, Perceptual image representation. EURASIP J. Image Video Process. 2007, 098181 (2007)

11. N Bruce, JK Tsotsos, Saliency based on information maximization. Adv. Neural Inform. Process. Syst. 18, 155-162 (2006)

12. W Luo, H Li, G Liu, KN Ngan, Global salient information maximization for saliency detection. Signal Process.: Image Commun. 27(3), 238-248 (2012)

13. J Harel, C Koch, P Perona, Graph-based visual saliency. Adv. Neural Inform. Process. Syst. 19, 545-552 (2006)

14. H Li, KN Ngan, A co-saliency model of image pairs. IEEE Trans. Image Process. 20(12), 3365-3375 (2011)

15. F Meng, H Li, G Liu, KN Ngan, Object co-segmentation based on shortest path algorithm and saliency model. IEEE Trans. Multimedia 14(5), 1429-1441 (2012)

16. H Li, F Meng, KN Ngan, Co-salient object detection from multiple images. IEEE Trans. Multimedia 15(8), 1896-1909 (2013)

17. T Liu, J Sun, NN Zheng, X Tang, HY Shum, Learning to detect a salient object, in Proceedings of IEEE Conference on Computer Vision and Pattern Recognition (CVPR) (Minneapolis, 18-23 June 2007), pp. 1-8

18. J Li, Y Tian, T Huang, W Gao, Multi-task rank learning for visual saliency estimation. IEEE Trans. Circuits Syst. Video Technol. 21(5), 623-636 (2011)

19. D Walther, C Koch, Modeling attention to salient proto-objects. Neural Netw. 19(9), 1395-1407 (2006)

20. R Achanta, F Estrada, P Wils, S Süsstrunk, Salient region detection and segmentation, in Proceedings of International Conference on Computer Vision Systems (ICVS), vol. 5008 (Santorini, 12-15 May 2008), pp. 66-75

21. R Achanta, S Hemami, F Estrada, S Süsstrunk, Frequency-tuned salient region detection, in Proceedings of IEEE Conference on Computer Vision and Pattern Recognition (CVPR) (Miami, 20-25 June 2009), pp. 1597-1604

22. MM Cheng, GX Zhang, NJ Mitra, X Huang, SM Hu, Global contrast based salient region detection, in Proceedings of IEEE Conference on Computer Vision and Pattern Recognition (CVPR) (Colorado Springs, 20-25 June 2011), pp. 409-416

23. T Judd, K Ehinger, F Durand, A Torralba, Learning to predict where humans look, in Proceedings of IEEE International Conference on Computer Vision (ICCV) (Kyoto, 27 Sept-4 Oct 2009), pp. 2106-2113
24. D Gao, N Vasconcelos, Decision-theoretic saliency: computational principles, biological plausibility, and implications for neurophysiology and psychophysics. Neural Comput. 21, 239-271 (2009)

25. KA Sundberg, JF Mitchell, JH Reynolds, Spatial attention modulates center-surround interactions in macaque visual area V4. Neuron 61(6), 952-963 (2009)

26. DH Kelly, Motion and vision.I. Stabilized images of stationary gratings. J. Opt. Soc. Am. 69, 1266-1274 (1979)

27. JR Cavanaugh, W Bair, JA Movshon, Nature and interaction of signals from the receptive field center and surround in macaque $\mathrm{V} 1$ neurons. J. Neurophysiol. 88(5), 2530-2546 (2002)

28. K Kerrmann, L Montaser-Kouhsari, M Carrasco, DJ Heeger, When size matters: attention affects performance by contrast or response gain. Nat. Neurosci. 13(12), 1554-1559 (2010)

29. L Xu, H Li, L Zeng, Z Wang, G Liu, Saliency detection using a central stimuli sensitivity based model, in Proceedings of IEEE International Symposium on Circuits and Systems (ISCAS) (Beijing, 19-23 May 2013), pp. 945-949

30. C Koch, S Ullman, Shifts in selective visual attention: towards the underlying neural circuitry. Hum. Neurobiol. 4, 219-227 (1985)

31. AM Treisman, G Gelade, A feature-integration theory of attention. Cogn. Psychol. 12, 97-136 (1980)

32. S Frintrop, E Rome, HI Christensen, Computational visual attention systems and their cognitive foundation: a survey. ACM Trans. Appl. Percept. 7, 6:1-6:39 (2010)

33. YF Ma, HJ Zhang, Contrast-based image attention analysis by using fuzzy growing, in Proceedings of ACM International Conference of Multimedia (Berkeley, 2-8 Nov 2003), pp. 374-381

34. R Achanta, S Süsstrunk, Saliency detection using maximum symmetric surround, in Proceedings of IEEE International Conference on Image Processing (ICIP) (Hong Kong, 26-29 Sept 2010), pp. 2653-2656

35. Y Hu, X Xie, W Ma, L Chia, D Rajan, Salient region detection using weighted feature maps based on the human visual attention model, in Proceedings of Fifth Pacific Rim Conference on Multimedia (Tokyo, 30 Nov-3 Dec 2004), pp. 993-1000

36. X Hou, L Zhang, Saliency detection: a spectral residual approach, in Proceedings of IEEE Conference on Computer Vision and Pattern Recognition (CVPR) (Minneapolis, 18-23 June 2007), pp. 1-8

37. Y Zhai, M Shah, Visual attention detection in video sequences using spatiotemporal cues, in Proceedings of ACM International Conference of Multimedia (Santa Barbara, 23-27 Oct 2006), pp. 815-824

38. F Perazzi, P Krähenbühl, Y Pritch, A Hornung, Saliency filters: contrast based filtering for salient region detection, in Proceedings of IEEE Conference on Computer Vision and Pattern Recognition (CVPR) (Providence, 16-21 June 2012), pp. 733-740

39. C Yang, L Zhang, H Lu, X Ruan, MH Yang, Saliency detection via graph-based manifold ranking, in Proceedings of IEEE Conference on Computer Vision and Pattern Recognition (CVPR) (Portland, 23-28 June 2013), pp. 3166-3173

40. X Li, H Lu, L Zhang, X Ruan, MH Yang, Saliency detection via dense and sparse reconstruction, in Proceedings of IEEE International Conference on Computer Vision (ICCV) (Sydney, 1-8 Dec 2013), pp. 2976-2983

41. W Wang, Y Wang, Q Huang, W Gao, Measuring visual saliency by site entropy rate, in Proceedings of IEEE Conference on Computer Vision and Pattern Recognition (CVPR) (San Francisco, 13-18 June 2010), pp. 2368-2375

42. L Itti, P Baldi, Bayesian surprise attracts human attention. Adv. Neural Inform. Process. Syst. 19, 547-554 (2006)

43. D Gao, N Vasconcelos, Bottom-up saliency is a discriminant process, in Proceedings of IEEE International Conference on Computer Vision (ICCV) (Rio de Janeiro, 14-20 Oct 2007), pp. 1-6

44. DA Klein, S Frintrop, Center-surround divergence of feature statistics for salient object detection, in Proceedings of IEEE International Conference on Computer Vision (ICCV) (Barcelona, 6-13 Nov 2011), pp. 2214-2219

45. L Zhang, MH Tong, TK Marks, H Shan, GW Cottrell, SUN: a Bayesian framework for saliency using natural statistics. J. Vis. 8(7), 1-20 (2008)

46. DR Vigo, J van de Weijer, T Gevers, Color edge saliency boosting using natural image statistics, in Proceedings of IS\&T's fifth European Conference on Colour in Graphics, Imaging, and Vision (CGIV) (Joensuu, 14-17 June 2010), pp. 228-234 
47. PN Belhumeur, JP Hespanha, DJ Kriegman, Eigenfaces Vs.Fisherfaces: recognition using class specific linear projection. IEEE Trans. Pattern Anal. Mach. Intell. 19(7), 711-720 (1997)

48. MP Sceniak, DL Ringach, MJ Hawken, R Shapley, Contrast's effect on spatial summation by macaque V1 neurons. Nat. Neurosci. 2(8), 733-739 (1999)

49. S Goferman, L Zelnik-Manor, A Talm, Context-aware saliency detection, in Proceedings of IEEE Conference on Computer Vision and Pattern Recognition (CVPR) (San Francisco, 13-18 June 2010), pp. 2376-2383

50. I van der Linde, U Rajashekar, AC Bovik, LK Cormack, DOVES: a database of visual eye movements. Spat. Vis. 22(2), 161-177 (2009)

doi:10.1186/1687-5281-2014-31

Cite this article as: Xu et al:: Saliency detection in complex scenes. EURASIP Journal on Image and Video Processing 2014 2014:31.

\section{Submit your manuscript to a SpringerOpen ${ }^{\circ}$ journal and benefit from:}

- Convenient online submission

- Rigorous peer review

- Immediate publication on acceptance

- Open access: articles freely available online

- High visibility within the field

- Retaining the copyright to your article 